Traditional Medicine and Modern Medicine

Vol. 1, No. 1 (2018) 43-51

(C) Institutes of Integrative Medicine, Fudan University and

World Century Publishing Corporation

DOI: $10.1142 /$ S2575900018500039

\title{
Urinary 8-hydroxydeoxyguanosine is a better biomarker of aging in non-smokers
}

\author{
Zhengxiao Zhao ${ }^{1,2}$, Weiyi Gong ${ }^{1,2}$, Lumei Liu ${ }^{1,2}$, Meng Wang ${ }^{3}$, Zihui Tang ${ }^{1,2}$, \\ Nabijan Mohammadtursun ${ }^{1,2}$, Linwei Lu ${ }^{1,2}$, Jiaqi Liu ${ }^{1,2}$, Mihui Li ${ }^{1,2}$, \\ Yibao $\mathrm{Lv}^{1,2}$, Shuming Mo ${ }^{1,2}$, Wenjuan $\mathrm{Ma}^{4}$ and Jingcheng Dong ${ }^{1,2, *}$ \\ ${ }^{1}$ Institute of Integrated Traditional Chinese and Western Medicine \\ Huashan Hospital, Fudan University \\ Shanghai, P. R. China \\ ${ }^{2}$ Institutes of Integrative Medicine, Fudan University \\ Shanghai, P. R. China \\ ${ }^{3}$ Physical Examination Center, Huashan Hospital \\ Fudan University, Shanghai, P. R. China \\ ${ }^{4}$ Department of Dermatology, Huashan Hospital \\ Fudan University, Shanghai, P. R. China \\ *jcdong2004@126.com
}

Received 18 July 2017; Accepted 1 December 2017; Published 2 February 2018

\begin{abstract}
Objective: The aim of this study is finding an optimal and convenient aging marker.
Method: The information of participants' occupation, family background, life style, physical condition, etc. was obtained by a self-administered questionnaire. Spot urine and saliva samples were collected during the early morning after fasting overnight. 8-hydroxydeoxygunaosine (8-OHdG), 8-iso-prostaglandin F2 $\alpha$ (8-isoPGF2 $\alpha$ ), advanced oxidation protein products (AOPPs), malondialdehyde (MDA), and dehydroepian drosterone sulfate (DHEA-S) were measured by ELISA.

Results: Around 112 non-smokers (males/females $=61 / 51$; mean age $=44.9$ years) were eligible and analyzed in this study. The mean urinary 8-OHdG level was $33.2 \pm 1.75$ (ng/mmol, creatinine) and mean 8-isoPGF2 $\alpha$ level was $364.97 \pm 40.32$ (pg/mmoL, creatinine). In saliva, the mean level of AOPPs, MDA, and DHEA-S was $189.37 \pm$ $19.81(\mu \mathrm{mol} / \mathrm{L}), 1.7 \pm 0.1(\mu \mathrm{mol} / \mathrm{L})$ and $2.96 \pm 0.15(\mathrm{ng} / \mathrm{mL})$, respectively. After analysis in multiple regression model, urinary 8-OHdG was influenced by age and tea consumption $(p<0.01)$; urinary 8-isoPGF2 $\alpha$ level was correlated with gender $(p<0.01)$; salivary DHEA-S was related to gender $(p<0.05)$ and marital status $(p<0.01)$; AOPPs and MDA in saliva presented no relationships with those factors.
\end{abstract}

Conclusion: Among healthy non-smokers, urinary 8-OHdG is the best aging biomarker comparing to other markers.

Keywords: 8-Hydroxydeoxyguanosine; urinary; saliva; aging biomarker; oxidative damage.

\section{Introduction}

Aging is characterized with homeostasis disorder and decrease of stress resistance. ${ }^{1}$ These physiological processes are critical risk factors of many diseases. Population aging is a severe challenge faced by the world at present. The soar of morbidity of geriatric diseases and the burden of medical costs highlight the importance of delaying aging or healthy aging. Butler et al. ${ }^{2}$ claimed anti-aging as a new model of health promotion and disease prevention for the 21 st century.

The aging process is highly individualized. Chronological age cannot represent the degree of aging. So, the primary task

\footnotetext{
*Corresponding author.
} 


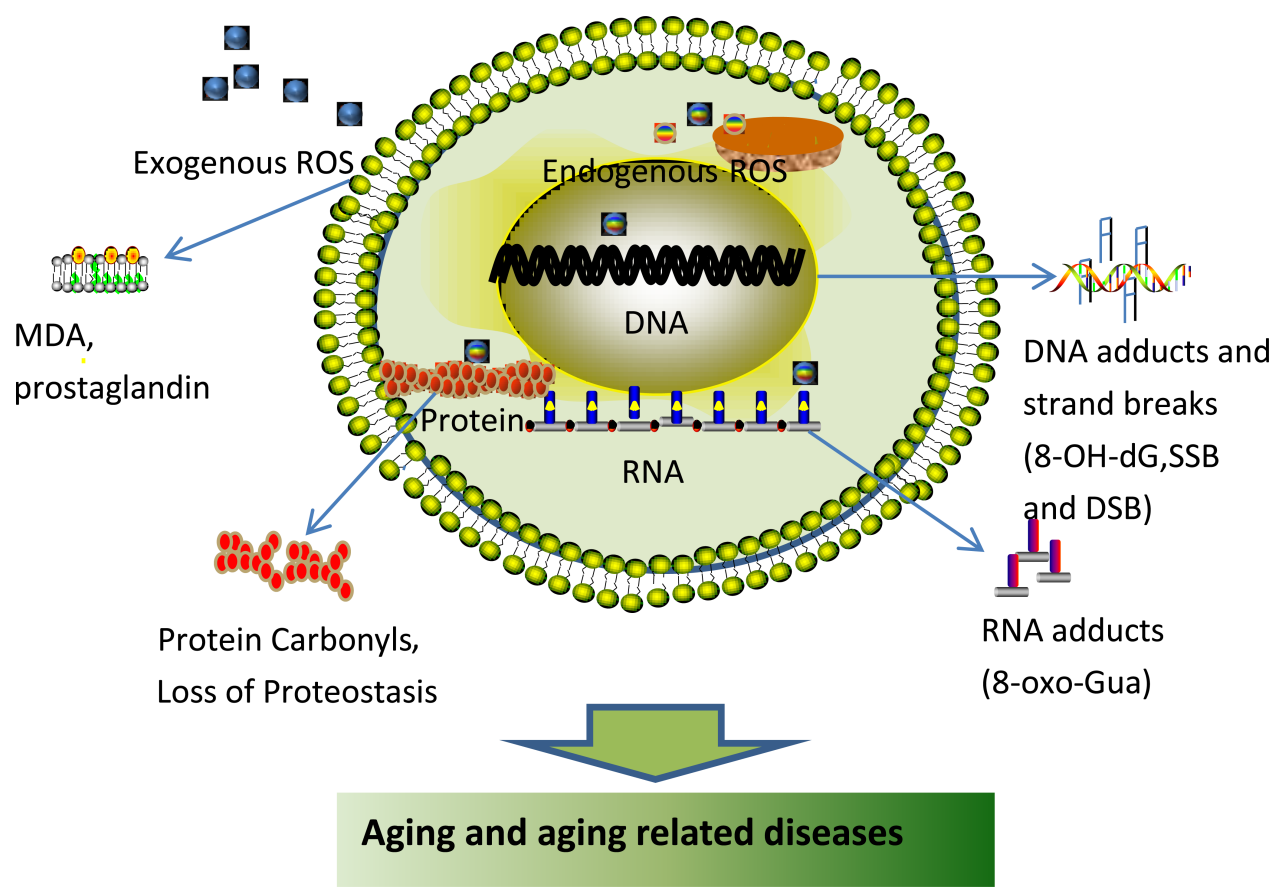

Figure 1. Oxidant stress in cell during aging. Various oxidative by-products or end-products are accumulated as a result of DNA, RNA, protein and lipid damage caused by ROS attack. This accumulation leads to aging and aging-related diseases.

is finding a simple aging marker besides life span to assess the biological age and evaluate the effectivity of anti-aging therapy. Research reported that a single cell is attacked by reactive oxygen species (ROS) $10^{3}$ to $10^{6}$ times per day which causes a progressive accumulation of oxidative damage in DNA, protein and lipid (Fig. 1). And it is a long-held hypothesis that biological aging is associated with the accumulation of these oxidative products which may account for aging-associated dysfunction in many biological processes. $^{3,4}$

Among these macromolecules, DNA is probably the most important target for ROS. ${ }^{5}$ And in DNA, the hydroxylation of guanine by ROS at the 8-position leads to G:C to T:A transversion, misreading of the modified base and mutagenesis. ${ }^{6}$ As it occurs, extensive and specific repair is performed by the cell for survival and to maintain genomic integrity, thus the product namely 8-hydroxydeoxyguanosine (8$\mathrm{OHdG}$ ) is excised and excreted to the urine. ${ }^{7}$ Many studies indicated that $8-\mathrm{OHdG}$ is a marker of oxidative damage, aging and some chronic diseases. However, as an aging marker, it is still controversial. Donati et al. ${ }^{8}$ found that the increased urinary output of $8-\mathrm{OHdG}$ mainly reflects the moment-to-moment activity of autophagy rather than aging. 8-iso-prostaglandin F2 $\alpha$ (8-isoPGF2 $\alpha$ ), a prostaglandin (PG) isomer derived from oxygen radical peroxidation of arachidonic acid esterified in cell membranes, ${ }^{8}$ destroys the integrity of cell membrane and has been regarded as a stable marker of cellular oxidative stress, ${ }^{9}$ but it is difficult to consistently define and specifically identify that markers of oxidative stress are directly linked to age and disease status. ${ }^{10}$ Malondialdehyde (MDA) is a famous product of peroxidation and reports showed that its concentration in elder's serum is higher than youngster's and it is widely used as an aging marker. ${ }^{11-14}$ Advanced oxidation protein products (AOPPs) are mainly derived from plasma albumin of oxidative modification resulting from the activity of ROS..$^{15}$ Researches reported that saliva AOPPs level in healthy adult increases with age, but some other studies demonstrated that saliva AOPPs were associated with caries rather than age. ${ }^{16}$ Dehydroepiandrosterone sulfate (DHEA-S) is the most abundant adrenal steroid in the blood. Its concentration in serum decreases with age and it is generally regarded as a reliable endocrine marker of aging.

In view of the miscellany and controversies of aging marker, we conducted a cross-sectional study which analyzed relationships of 8 -OHdG, 8 -isoPGF2 $\alpha$ in human urine and AOPPs, MDA, DHEA-S in human saliva with age and influence of various lifestyle factors by using multivariate regression analysis. For the first time, we compared these compounds in urine and saliva in order to find out which one is better.

\section{Subjects and Methods}

\section{Design and subjects}

After approval by the ethics committee of Huashan Hospital, Fudan University, participants were recruited from physical 
examination center of Huashan Hospital through announcements asking for volunteers to join in the study. After obtaining the written informed consent from the participants, information about their occupation, family background, life style, etc. was collected by a self-administered questionnaire. Items included in the questionnaire were age, gender, residence, education, marital state, tea/alcohol/cigarettes consumption, present illness, past medical history, family background and preference of their diet. According to the age division criteria of $\mathrm{WHO}$, we divided the participants into three groups (young $(<45)$, middle age $(45-59)$ and old $(>60))$. Education attainment was divided into three levels: well (master degree or higher), moderate (college degree), poorly (high school degree and lower). The frequency of green tea intake was assessed according to the following three ranked options: almost every day (often), two to three times a week (occasional), less than once a week (never). Alcohol/cigarettes consumption was presented as yes or no. The diet habit was defined according to the meat or vegetables consumption. Waistline was measured by tape. Blood pressure of all subjects was measured by a sphygmomanometer. Body mass index (BMI) was calculated as weight/ (height $*$ height). Inclusion criteria are as follows: (1) Person who lives in Shanghai and nearby regions. (2) Non-smokers aged above 20 years old. (3) Having no disease history and severe alcohol drinking habit. Exclusion criteria are as follows: (1) Participants who have oral infection and urinary tract infection. (2) People who are currently taking antioxidant or other drugs. (3) People who have cognition disorder. (4) Pregnant women. (5) People who have abnormal results of their blood routine examination, liver function, renal function, electrocardiogram and chest fluoroscopy.

\section{Materials and methods}

\section{Sample collection}

Saliva samples were collected after fasting from 22:00 the day before by using salivettes (Sarstedt, Germany). Specifically, participants were asked to gargle for three times using water and take a $5 \mathrm{~min}$ rest before collecting. Then, the sterile cotton column in salivettes was given to participants and chewed for 5 min and spat into the tube. After that, the salivette was centrifuged for $10 \mathrm{~min}\left(4^{\circ} \mathrm{C}, 3000 \mathrm{rpm}\right)$ and saliva was pipetted into another tube. Samples were stored at $-80^{\circ} \mathrm{C}$ until test. First morning, urine samples were collected in $50 \mathrm{~mL}$ polypropylene tubes and stored at $-80^{\circ} \mathrm{C}$ until analysis.

\section{Analysis of urinary 8-OHdG}

The level of urinary 8-OHdG was determined by an enzyme immunoassay (EIA) kit (Stressmarq, Canada). In brief, the bulk standard $(30 \mathrm{ng} / \mathrm{mL})$ was gradient diluted into eight tubes. About $100 \mu \mathrm{L}$ EIA Buffer was added to Non-Specific Binding wells and $50 \mu \mathrm{L}$ EIA Buffer to Maximum Binding wells. About $50 \mu \mathrm{L}$ standard $(10.3 \mathrm{pg} / \mathrm{mL}-30 \mathrm{ng} / \mathrm{mL})$ from each tube was added to the standard wells orderly until all the eight standards are aliquoted. About $50 \mu \mathrm{L}$ of sample and 8OHdG AChE Tracer (except the Total Activity and the Blank wells) and 8-OHdG Monoclonal Antibody (except the Total Activity, the Non-Specific Binding well) were added to each well. Each sample was assayed at a minimum of two dilutions and was assayed in triplicate. The Plate was incubated for $18 \mathrm{~h}$ at $4^{\circ} \mathrm{C}$. The wells were emptied and rinsed for five times with Wash Buffer. Add $200 \mu \mathrm{L}$ of Ellman's Reagent to each well. Add $5 \mu \mathrm{L}$ of tracer to the Total Activity wells. Optimum development is obtained by using an orbital shaker equipped with a large, flat cover to allow the plate to develop in the dark. This assay typically develops in 90-120 min. Absorbance of each well was read at $420 \mathrm{~nm}$ by a microplate reader.

\section{Analysis of urinary 8-isoPGF2 $\boldsymbol{\alpha}$}

The urine samples were analyzed for 8 -isoPGF $2 \alpha$ levels by a competitive enzyme-linked immunoassay (ELISA) kit (Enzo biochem, USA). The kit used a polyclonal antibody to bind, in a competitive manner, 8 -isoPGF $2 \alpha$ in a sample or standard (6.1-100,000 pg/mL). After a simultaneous incubation at room temperature, the excess reagents were washed away and substrate was added. After a short incubation time, the enzyme reaction was stopped and the yellow color generated was read on a microplate reader at $405 \mathrm{~nm}$.

\section{Analysis of salivary AOPPS}

Saliva AOPPs were assayed by an OxiSelect ${ }^{\mathrm{TM}}$ AOPP Assay Kit. Samples were prepared according to the protocol. About $200 \mu \mathrm{L}$ of samples or standards $(0-250 \mu \mathrm{M})$ were added to separate wells of the microtiter plate and $10 \mu \mathrm{L}$ of Chloramine Reaction Initiator to each well. Then, samples and reagents were mixed thoroughly and incubated on a table top rotator or shaker for $5 \mathrm{~min}$. About $20 \mu \mathrm{L}$ of Stop Solution was added to each well. Absorbance was read immediately on a spectrophotometric plate reader using $340 \mathrm{~nm}$ as the primary wave length and $0 \mu \mathrm{M}$ Chloramine standard was used as an absorbance blank.

\section{Analysis of salivary MDA}

MDA was assayed by a commercially available ELISA kit (Oxford biomedical research, USA). Briefly, samples were prepared following the protocol, $200 \mu \mathrm{L}$ standard $(0-20 \mu \mathrm{M})$ or sample and $200 \mu \mathrm{L}$ Indicator Solution was added into each 
well, and then incubated at room temperature for $45 \mathrm{~min}$. About $150 \mu \mathrm{L}$ of each solution was transferred to the microplate and read at $540 \mathrm{~nm}$.

\section{Analysis of salivary DHEA-S}

Saliva DHEA-S was assayed by ELISA kit (LDN, Germany). In brief, $50 \mu \mathrm{L}$ of standard $(0-12 \mathrm{ng} / \mathrm{mL})$ or sample and $150 \mu \mathrm{L}$ diluted conjugate was added to the wells. The plate was incubated at $37^{\circ} \mathrm{C}$ for $15 \mathrm{~min}$. Contents of each well were removed. The wells were washed for three times with $0.3 \mathrm{~mL}$ of diluted wash solution. During each washing step, the plate was gently shaken for $5 \mathrm{~s}$ and excess solution was removed by tapping the inverted plate on an absorbent paper

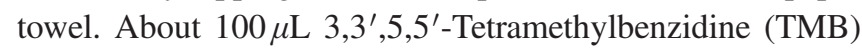
Substrate was added. After incubating at room temperature $\left(22-28^{\circ} \mathrm{C}\right)$ for $15 \mathrm{~min}$ in the dark, $100 \mu \mathrm{L}$ of stop solution was added into the wells. Absorbance $(E)$ was read at $450 \mathrm{~nm}$.

\section{Analysis of creatinine}

Creatinine was assayed by enzymic creatinine assay method. Briefly, $6 \mu \mathrm{L}$ of sample or standard and $180 \mu \mathrm{L}$ of enzyme solution was added to each well. Then, plate was incubated at $37^{\circ} \mathrm{C}$ for $5 \mathrm{~min}$ and read the absorbance at $546 \mathrm{~nm}$.

\section{Statistical analysis}

All data were analyzed using the SPSS 20.0 statistical package (IBM, USA) for Windows. Mean \pm SEM were used for descriptive statistics. The relationship between host factors and the biomarkers' levels was analyzed by using one-way ANOVA or non-parametric Kruscal-Wallis test according to the distribution of the data while the relationship between BMI, waistline, systolic pressure (SP), diastolic pressure (DP) and the biomarkers' levels was analyzed by using Spearman's rank correlation. A multiple regression analysis using a stepwise method was then performed to evaluate how these markers' levels as the dependent variable was determined by the independent variables. $p<0.05$ was accepted as indicating statistical significance.

\section{Results}

Finally, 112 healthy subjects (61 males and 51 females) were eligible. Demographic and lifestyle characteristics of the study population were showed in Table 1. Data revealed that mean age was $44.9( \pm 15.1)$ years old (range 23-83). Nearly, half of the subjects graduated from college $(55.5 \%)$ and most of them got married (82.1\%). No significant differences were seen in the tea consumption, diet preference and family background (life span of the elders).

Table 1. Demographic and lifestyle characteristics of 112 healthy participants.

\begin{tabular}{llcc}
\hline Variables & Category & $N$ & Percentage (\%) \\
\hline Sex & Male & 61 & 54.5 \\
Age & Female & 51 & 45.5 \\
& Young (20-44) & 61 & 54.5 \\
Education attainment & Middle-aged (45-59) & 27 & 24.1 \\
& Old (60-) & 24 & 21.4 \\
& Mell & 18 & 16.4 \\
Marital status & Moderate & 61 & 55.5 \\
Tea consumption & Married & 31 & 28.2 \\
& Unmarried & 92 & 82.1 \\
\multirow{2}{*}{ Diet preference } & Often & 20 & 17.9 \\
& Necasional & 43 & 38.4 \\
Family background & Vegetarian diet & 45 & 40.2 \\
& High fat and protein food & 24 & 21.4 \\
& No preference & 44 & 9.3 \\
Variables & AM & 31 & 33 \\
\hline BMI & & 52 & 27 \\
BP & & 60 & 46.4 \\
Waistline & & Mean & 53.6 \\
\hline
\end{tabular}

Notes: BMI: body mass index; BP: blood pressure; NO: nonagenarian offspring; AM: age-matched control. 
Table 2. Association of 8-OHdG, 8-isoPGF2 $\alpha$, AOPPs, MDA and DHEA-S with host factors.

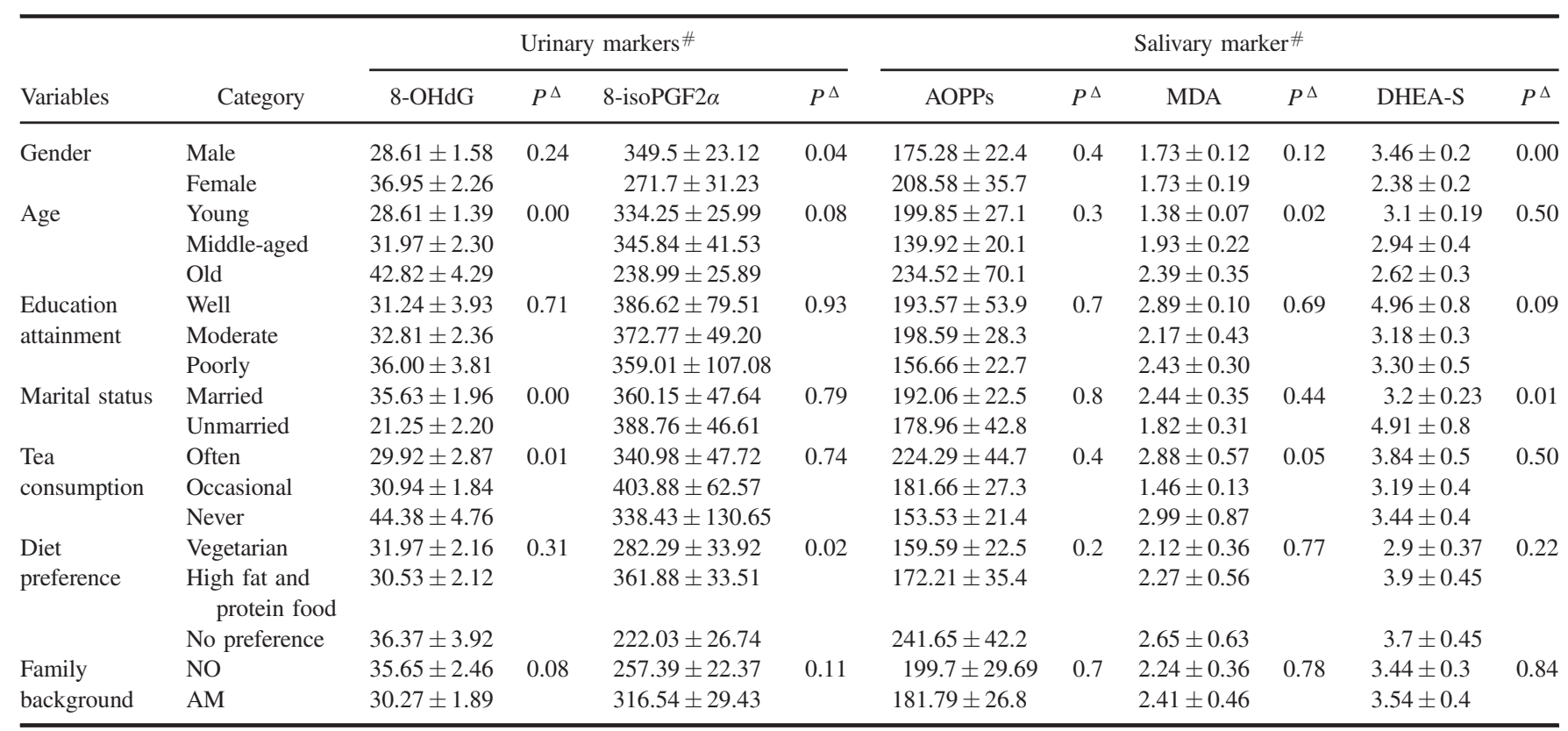

Notes: ${ }^{\triangle} P$-value is obtained by one-way analysis of variance. NO: nonagenarian offspring; AM: age-matched control. \# Data are Mean $\pm \mathrm{SE}: 8-\mathrm{OHdG}$ (ng/mmol, creatinine); 8 -isoPGF2 $\alpha$ (pg/mmoL, creatinine); AOPPs $(\mu \mathrm{mol} / \mathrm{L})$; MDA $(\mu \mathrm{mol} / \mathrm{L})$; DHEA-S $(\mathrm{ng} / \mathrm{mL})$.

With the aim of screening out the better aging marker from these markers, we investigated the association of 8-OHdG, 8-isoPGF2 $\alpha$, AOPPs, MDA and DHEA-S with host factors including gender, age, education attainment, marital status, tea consumption, diet preference and family background (Table 2) or BMI, waistline, SP and DP (Table 3). Then, variables were selected using a stepwise selection procedure with the level of entry determined according to a multiple regression analysis of 8-OHdG, 8 -isoPGF2 $\alpha$, MDA and DHEA-S (Table 4). The mean urinary $8-\mathrm{OHdG}$ level was $33.2 \pm 1.75$ (ng/mmol, creatinine) in 112 non-smokers. Univariate analysis showed that $8-\mathrm{OHdG}$ increased with age and the difference was statistically significant $(p<0.01)$. Marital status and tea consumption were also correlated with 8-OHdG, but influence of marital status was excluded in multiple regression analysis. Results showed that drinking tea reduced $8-\mathrm{OHdG}$ content in urine. The mean 8 -isoPGF $2 \alpha$ level was $364.97 \pm 40.32(\mathrm{pg} / \mathrm{mmoL}$, creatinine). 8-isoPGF $2 \alpha$ was related to gender and diet preference in univariate analysis. Male or people preferring to high fat and protein food had higher concentration of 8-isoPGF2 $\alpha$ in their urine. In multivariate models, no association between diet preference and 8-isoPGF $2 \alpha$ was observed. As to AOPPs in saliva, neither univariate analysis nor multiple regression analysis presented statistically significant changes. Univariate analysis indicated that MDA was positively correlated with age $(r=0.203, p<0.05)$. In addition, MDA was positively related to waistline $(r=0.209, p<0.05)$ and negatively correlated with SP $(r=-0.247, p<0.01)$. But, only association between MDA and waistline was statistically significant in multivariable-adjusted analysis. Unlike the oxidative markers, endocrine marker, salivary DHEA-S decreased with age, but no statistical significance was observed in our study. The mean level of DHEA-S of all participants was $2.96 \pm$ $0.15(\mathrm{ng} / \mathrm{mL})$. According to univariate analysis, gender, marital status, BMI, waistline and SP significantly influenced the level of DHEA-S. While analyzed in the multivariable regression model, the effects of BMI, waistline and SP were

Table 3. Association of 8-OHdG, 8-isoPGF2 $\alpha$, AOPPs, MDA and DHEA-S with BMI, Waistline, SP and DP.

\begin{tabular}{|c|c|c|c|c|c|c|c|c|c|c|c|}
\hline \multirow[b]{2}{*}{ Variables } & \multirow[b]{2}{*}{ Mean $\pm \mathrm{SE}$} & \multicolumn{4}{|c|}{ Urinary markers } & \multicolumn{6}{|c|}{ Salivary marker } \\
\hline & & 8-OHdG $(r)$ & $P$ & 8 -isoPGF2 $\alpha(r)$ & $P$ & $\operatorname{AOPPs}(r)$ & $P$ & $\operatorname{MDA}(r)$ & $P$ & DHEA-S $(r)$ & $P$ \\
\hline BMI & $23.11 \pm 0.3$ & -0.007 & 0.95 & -0.103 & 0.34 & -0.036 & 0.76 & 0.165 & 0.09 & 0.273 & 0.01 \\
\hline Waistline & $80.47 \pm 1.0$ & 0.009 & 0.93 & 0.042 & 0.71 & -0.112 & 0.35 & 0.209 & 0.05 & 0.328 & 0.00 \\
\hline SP & $117.83 \pm 1.3$ & 0.106 & 0.31 & 0.032 & 0.77 & 0.008 & 0.94 & -0.247 & 0.01 & -0.211 & 0.03 \\
\hline DP & $74.85 \pm 0.9$ & 0.026 & 0.81 & -0.024 & 0.82 & -0.002 & 0.98 & -0.130 & 0.19 & -0.160 & 0.11 \\
\hline
\end{tabular}

Notes: BMI: body mass index; SP: systolic pressure; DP: diastolic pressure. 
Table 4. Multiple regression analysis of 8-OHdG, 8 -isoPGF2 $\alpha$, MDA and DHEA-S.

\begin{tabular}{|c|c|c|c|c|c|c|}
\hline \multicolumn{7}{|c|}{ 8-OHdG } \\
\hline Variable & Coef. & Std. Err. & $t$ & $P$ & 95\% Conf. & Interval \\
\hline Age & 0.24 & 0.10 & 2.52 & 0.013 & {$[0.05$} & $0.43]$ \\
\hline Tea consumption & 7.58 & 1.78 & 4.27 & 0.000 & {$[4.05$} & 11.11] \\
\hline Marital status & -7.17 & 4.02 & -1.79 & 0.078 & {$[-15.05$} & $0.71]$ \\
\hline \multicolumn{7}{|l|}{ 8-isoPGF2 $\alpha$} \\
\hline Gender & -83.37 & 38.66 & -2.16 & 0.034 & {$[-160.37$} & $-6.37]$ \\
\hline Diet preference & -34.14 & 22.59 & -1.51 & 0.134 & {$[-78.42$} & $10.14]$ \\
\hline \multicolumn{7}{|l|}{ MDA } \\
\hline Age & 0.55 & 0.46 & 1.19 & 0.239 & {$[-037$} & $1.48]$ \\
\hline Waistline & 0.7 & 0.03 & 2.194 & 0.032 & {$[0.01$} & $0.13]$ \\
\hline SP & -0.03 & 0.03 & -1.14 & 0.259 & {$[-0.08$} & $0.02]$ \\
\hline \multicolumn{7}{|l|}{ DHEA-S } \\
\hline Gender & -1.147 & 0.56 & -2.05 & 0.044 & {$[-2.26$} & $-0.03]$ \\
\hline Marital status & 0.277 & 0.09 & 2.95 & 0.004 & {$[0.09$} & $0.46]$ \\
\hline BMI & 0.230 & 0.16 & 1.39 & 0.167 & {$[-0.06$} & $0.60]$ \\
\hline Waistline & 0.018 & 0.06 & 0.32 & 0.75 & {$[-0.09$} & $0.13]$ \\
\hline SP & -0.010 & 0.02 & -0.40 & 0.690 & {$[-0.05$} & $0.03]$ \\
\hline
\end{tabular}

Notes: Statistical analysis was conducted by a stepwise multiple regression analysis. Coef. (regression coefficient); Std. Err. (standard error); 95\% Conf. Interval (95\% confidence interval).

ruled out. Our study indicated that male had about 1.45 folds higher DHEA-S level than female $(p<0.01)$. Comparing with the married people, salivary DHEA-S level in unmarried young people increased 1.5 folds $(p<0.01)$.

\section{Discussion}

Appropriate biomarkers which can properly evaluate the biological age and the effect of anti-aging therapy play a critical role in healthy aging. Gerontologists have devoted themselves to looking for aging biomarkers for decades. ${ }^{17}$ The existed aging markers mostly emerged from animal models with a complicated analytic method. ${ }^{18}$ In this paper, we analyzed and compared how the humans' urinary 8OHdG, 8 -isoPGF2 $\alpha$ and salivary AOPPs, MDA and DHEA-S levels were related to various factors using multiple regression analysis method and with the purpose of finding a convenient, appropriate, economical and practical aging marker.

In the univariate analysis, we found that $8-\mathrm{OHdG}$ was related to age, tea consumption and marital status. Marital status was excluded in multiple regression analysis. The positive association with age is consistent with other studies. ${ }^{19,20}$ Noren et $a l .{ }^{21}$ also obtained the result of positive correlation between $8-\mathrm{OHdG}$ and age by measuring plasma 8-OHdG using ELISA. With the increase of age, the chance that subjects exposing to exogenous detrimental substances like radiation, drug, alcohol or metal element increases. ROS production increases because of the decline of mitochondrial function and dysfunction of free radical scavenging system and antioxidant system. These all result in the accumulation of DNA oxidative damage and increase of its product, 8-OHdG. In our study, 8-OHdG decreased with the increasing amount of tea consumption and this may relate to the tea polyphenol components. A large amount of researches indicated that tea polyphenol has significant antioxidant effects. $^{22-24}$ It is an anti-aging ingredient which can prolong the life span of nematode, drosophila and other animal models. ${ }^{25-27}$ These results showed that $8-\mathrm{OHdG}$ is an appropriate and convenient aging biomarker which can be widely used in anti-aging medicine. With regard to family background, nonagenarian offspring has a higher 8-OHdG concentration than their age-matched control. This is opposite to our expectation. Probably, it is because that we did not examine the longevity genes, and the offspring of long-lived people could not be guaranteed to inherit the longevity genes. Inevitably, experimental error could not be excluded, either.

In terms of urinary 8 -isoPGF $2 \alpha$, our study found that there was no association between urinary 8 -isoPGF $2 \alpha$ and age. This result is consistent with other relevant reports indicating that the content of 8 -isoPGF $2 \alpha$ released by rat brain synaptosomes was independent from aging in the basal state. ${ }^{28}$ But, a previous study had shown that the average level of esterified form 8-isoPGF2 $\alpha$ in the plasma from aged rats was 30.6-fold higher than that in the plasma from young rats. ${ }^{29}$ Different species and samples examined sites may be the reason of this discrepancy. The multivariate analysis showed that urinary 8 -isoPGF $2 \alpha$ level of male is significantly higher than that in female. It means lipid peroxidation of male is more severe than female. This may be related to the 
influence by sex hormones to the lipid metabolism, ${ }^{30-32}$ study indicated that the decrease in estrogen level leads to an increase of lipid peroxidation, a decrease in the incidence of metabolism syndrome was observed in women treated with hormone therapy (estradiol, progestin), and this may related to the increased triglyceride and HDL-c levels. ${ }^{33}$ Our results showed that urinary 8 -isoPGF $2 \alpha$ of non-smokers cannot be an aging marker.

AOPPs had no association to age or other variables in our study. This is contradictory to Zeng's ${ }^{15}$ results which obtained a positive correlation with age. However, there is a new report which confirmed that the influence of age to AOPPs was little and the main influencing factor was dental caries. $^{34}$ These conflicting reports may be due to unclear source, function and composition of AOPPs. Based on our result, AOPPs cannot be an aging marker.

MDA in blood or tissue has been regarded as biomarkers of many oxidation-related diseases such as coronary heart disease, diabetes, cancer and senescence. ${ }^{11,35,36}$ Many studies reported an age-dependent increase of MDA. ${ }^{12,37}$ In the univariate analysis of our study, we have obtained a similar result. In addition, we found that MDA was influenced by waistline and SP in univariate analysis. When the three factors were analyzed in multivariate regression model, the significant difference was only observed between waistline and MDA. Therefore, MDA in saliva cannot be an aging marker.

DHEA-S exerts many biological activities including stimulating neurite growth, neurogenesis and neuronal survival, apoptosis, catecholamine synthesis and secretion, together with antioxidant, anti-inflammatory and antiglucocorticoid properties. ${ }^{38}$ Study showed that serum DHEA-S steadily decreased with age by $10 \%$ per decade reaching a nadir after the age of $80 .{ }^{39,40}$ Our study indicated that salivary DHEA-S decreased with age, but there was no significant difference. Research showed that the reliability of using DHEA-S as a biomarker of aging may be compromised in calorie restriction studies. ${ }^{41}$ Dietary manipulations may affect liver enzymes involved in the metabolism of steroid hormones. The result in our study may also be related to the small sample size or less age apart. When we divided the participants into six groups and each group was 10 years apart, the difference between the youngest group (20-30 years) and the eldest group (70 years above) was significant $(p<0.05)$. Gender, marital status, BMI, waistline and SP were associated with DHEA$\mathrm{S}$ in univariate analysis, but when adjusted in the regression model, DHEA-S was only influenced by gender and marital status. Being coincident with other studies, concentration of male salivary DHEA-S was significantly higher than female in our study. The different levels of sex-steroid hormones between adult males and females may play a contributing role. ${ }^{42}$ Salivary DHEA-S was lower in married people in our study. However, there is no study showing the influence of marital status to DHEA-S. The working pressure or the elder age of married people may be the reason of this lower level of DHEA-S.

\section{Conclusion}

We conducted a cross-sectional study in which 112 healthy non-smokers were enrolled. We firstly analyzed and compared the relationship between five potential aging markers (8-OHdG, 8-isoPGF2 $\alpha$, AOPPs, MDA and DHEA-S) in the urine or saliva and lifestyles or individual situations by using multiple regression models. We found that in non-smokers, urinary $8-\mathrm{OHdG}$ is the optimal aging marker comparing to other markers. This provided great value in evaluation of aging. It avoids the wrong choice among multifarious aging biomarkers and can be further used in clinical practice. But, cross-sectional study limits the explanation of the results and recall bias exists in the self-administrated investigation. Therefore, a prospective study in the future is needed.

\section{Conflict of Interest}

The authors report no conflict of interest in association with this work.

\section{Acknowledgments}

We would like to thank Zhengsheng $\mathrm{Xu}$ and Yihui Guan for providing convenience and kind help. This work was partly supported by the National Science Foundation for Distinguished Young Scholars of China (Grant No. 81403148) and Development Project of Shanghai Peak Disciplines-Integrative Medicine (Grant No. 20150407).

\section{References}

1. Lopez-Otin C, Blasco MA, Partridge L, et al. The hallmarks of aging. Cell 2013;153:1194-217.

2. Butler RN, Miller RA, Perry D, et al. New model of health promotion and disease prevention for the 21 st century. BMJ 2008;337:a399.

3. Donati A, Cavallini G, Bergamini E. Effects of aging, antiaging calorie restriction and in vivo stimulation of autophagy on the urinary excretion of $8 \mathrm{OHdG}$ in male Sprague-Dawley rats. Age 2013;35:261-70.

4. Gruber J, Schaffer S, Halliwell B. The mitochondrial free radical theory of ageing-where do we stand? Front Biosci 2008;13:6554-79.

5. Schieber M, Chandel NS. ROS function in redox signaling and oxidative stress. Curr Biol 2014;24:R453.

6. Shen J, Deininger P, Hunt JD, et al. 8-Hydroxy-2'-deoxyguanosine $(8-\mathrm{OH}-\mathrm{dG})$ as a potential survival biomarker 
in patients with nonsmall-cell lung cancer. Cancer 2007; 109:574-80.

7. Chiou C, Chang P, Chan E, et al. Urinary 8-hydroxydeoxyguanosine and its analogs as DNA marker of oxidative stress: Development of an ELISA and measurement in both bladder and prostate cancers. Clin Chim Acta 2003;334:87-94.

8. Wood LG, Gibson PG, Garg ML. Biomarkers of lipid peroxidation, airway inflammation and asthma. Eur Respir J 2003;21:177.

9. Tacconelli S, Capone ML, Patrignani P. Measurement of 8-isoprostaglandin F2alpha in biological fluids as a measure of lipid peroxidation. Methods Mol Biol 2010;644:165-78.

10. Jacob KD, Noren HN, Trzeciak AR, et al. Markers of oxidant stress that are clinically relevant in aging and age-related disease. Mech Ageing Dev 2013;134:139-57.

11. Gil P, Farinas F, Casado A, et al. Malondialdehyde: A possible marker of ageing. Gerontology 2002;48:209-14.

12. Inal ME, Kanbak G, Sunal E. Antioxidant enzyme activities and malondialdehyde levels related to aging. Clin Chim Acta 2001;305:75-80.

13. Mutlu-Turkoglu U, Ilhan E, Oztezcan S, et al. Age-related increases in plasma malondialdehyde and protein carbonyl levels and lymphocyte DNA damage in elderly subjects. Clin Biochem 2003;36:397-400.

14. Traverso N, Patriarca S, Balbis E, et al. Anti malondialdehydeadduct immunological response as a possible marker of successful aging. Exp Gerontol 2003;38:1129-35.

15. Qing Z, Ling-Ling E, Wang DS, et al. Relationship of advanced oxidative protein products in human saliva and plasma: Age- and gender-related changes and stability during storage. Free Radical Res 2012;46:1201-6.

16. Celecova V, Kamodyova N, Tothova L, et al. Salivary markers of oxidative stress are related to age and oral health in adult non-smokers. J Oral Pathol Med 2013;42:263-6.

17. Vidal EI, Mayoral VF, Villas BP, et al. Physical frailty as a clinical marker of biological age and aging. J Am Geriatr Soc 2015;63:837-8.

18. Engelfriet PM, Jansen EH, Picavet HS, et al. Biochemical markers of aging for longitudinal studies in humans. Epidemiol Rev 2013;35:132-51.

19. Li YS, Song MF, Kasai H, et al. 8-hydroxyguanine in urine and serum as an oxidative stress marker: Effects of diabetes and aging. J Uoeh 2013;35:119.

20. Siomek A, Gackowski D, Rozalski R. Higher leukocyte 8-oxo7,8-dihydro-20-deoxyguanosine and lower plasma ascorbate in aging humans? Antioxid Redox Sign 2007;9:143-50.

21. Noren HN, Ejiogu N, Zonderman AB, et al. Association of oxidative DNA damage and C-reactive protein in women at risk for cardiovascular disease. Arterioscler Thromb Vasc Biol 2012;32:2776-84.

22. Park YH, Han DW, Suh H, et al. Protective effects of green tea polyphenol against reactive oxygen species-induced oxidative stress in cultured rat calvarial osteoblast. Cell Biol Toxicol 2003;19:325-37.

23. Rah DK, Han DW, Baek HS, et al. Prevention of reactive oxygen species-induced oxidative stress in human microvascular endothelial cells by green tea polyphenol. Toxicol Lett 2005; 155:269-75.

24. Cavet ME, Harrington KL, Vollmer TR, et al. Anti-inflammatory and anti-oxidative effects of the green tea polyphenol epigallocatechin gallate in human corneal epithelial cells. Mol Vis 2011;17:533-42.

25. Kitani K, Osawa T, Yokozawa T. The effects of tetrahydrocurcumin and green tea polyphenol on the survival of male C57BL/6 mice. Biogerontology 2007;8:567-73.

26. Abbas S, Wink M. Epigallocatechin gallate from green tea (Camellia sinensis) increases lifespan and stress resistance in Caenorhabditis elegans. Planta Med 2009;75:216-21.

27. Lopez T, Schriner SE, Okoro M, et al. Green tea polyphenols extend the lifespan of male drosophila melanogaster while impairing reproductive fitness. J Med Food 2014; 17:1314-21.

28. Brunetti L, Michelotto B, Orlando G, et al. Aging increases amyloid beta-peptide-induced 8-iso-prostaglandin F2alpha release from rat brain. Neurobiol Aging 2004;25:125-9.

29. Chu X, Ageishi Y, Nishimura K, et al. Development of enzyme-linked immunosorbent assay for 8-iso-prostaglandin F2alpha, a biomarker of oxidative stress in vivo, and its application to the quantification in aged rats. J Pharm Biomed Anal 2009;50:911-6.

30. Morrison JA, Sprecher DL, Biro FM, et al. Serum testosterone associates with lower high-density lipoprotein cholesterol in black and white males, 10 to 15 years of age, through lowered apolipoprotein AI and AII concentrations. Metabolism 2002;51:432-7.

31. Morrison JA, Barton BA, Biro FM, et al. Sex hormones and the changes in adolescent male lipids: Longitudinal studies in a biracial cohort. J Pediatr 2003;142:637-42.

32. Van Pottelbergh I, Braeckman L, De Bacquer D, et al. Differential contribution of testosterone and estradiol in the determination of cholesterol and lipoprotein profile in healthy middle-aged men. Atherosclerosis 2003;166:95-102.

33. Sanchez-Rodriguez MA, Zacarias-Flores M, Castrejon-Delgado L, et al. Effects of hormone therapy on oxidative stress in postmenopausal women with metabolic syndrome. Int $\mathrm{J}$ Mol Sci 2016;17(9):13-88.

34. Venturini D, Simao AN, Dichi I. Advanced oxidation protein products are more related to metabolic syndrome components than biomarkers of lipid peroxidation. Nutr Res 2015; 35:759-65.

35. Olszewska-Slonina DM, Matewski D, Czajkowski R, et al. The concentration of thiobarbituric acid reactive substances (TBARS) and paraoxonase activity in blood of patients with osteoarthrosis after endoprosthesis implantation. Med Sci Monit 2011;17:498-504.

36. Lee R, Antoniades C. Thiobarbituric acid reactive substances as a biomarker for coronary heart disease. J Atheroscler Thromb 2011;18:1127-8.

37. Kawamoto EM, Vasconcelos AR, Degaspari S, et al. Agerelated changes in nitric oxide activity, cyclic GMP, and TBARS levels in platelets and erythrocytes reflect the oxidative status in central nervous system. Age 2013;35:331-42.

38. Maggio M, De Vita F, Fisichella A, et al. DHEA and cognitive function in the elderly. J Steroid Biochem Mol Biol 2015; 145:281-92.

39. Tivesten A, Vandenput L, Carlzon D, et al. Dehydroepiandrosterone and its sulfate predict the 5-year risk of coronary heart disease events in elderly men. J Am Coll Cardiol 2014;64:1801. 
40. Traish AM, Kang HP, Saad F, et al. Dehydroepiandrosterone (DHEA) - a precursor steroid or an active hormone in human physiology. J Sex Med 2011;8:2960-83.

41. Urbanski HF, Mattison JA, Roth GS, et al. Dehydroepiandrosterone sulfate (DHEAS) as an endocrine marker of aging in calorie restriction studies. Exp Gerontol 2013; 48:1136-9.

42. Sorwell KG, Kohama SG, Urbanski HF. Testosterone increases circulating dehydroepiandrosterone sulfate levels in the male rhesus macaque. Front Endocrinol (Lausanne) 2014;5:101 